\title{
The nutrient composition of South African lamb (A2 grade)
}

\author{
S.M. van Heerden ${ }^{a}$, H.C. Schönfeldt ${ }^{b}$, R. Kruger ${ }^{b}$ and M.F. Smit ${ }^{c}$ \\ aRC-LBD: Animal Production, ARC-IRENE, Private Bag X2, Irene 0062, South Africa \\ ${ }^{b}$ Department of Business Economics, University of Pretoria, Pretoria 0002, South Africa \\ ${ }^{\mathrm{c}}$ ARC-Biometry Unit, Private Bag X519, Silverton 0127, South Africa
}

\begin{abstract}
Dorper and Merino lamb carcasses of the A age group with a fat code 2 ( $\pm 7 \%$ SCF) from three main production areas in South Africa were used for this study. The right sides of the carcasses were used to determine the raw nutrient and physical (carcass) composition of each cut as well as for the whole carcass by calculation. Three cuts (shoulder, loin and leg) from the left side were cooked in order to determine the nutrient composition thereof. Nutrients showing the greatest differences between raw and cooked treatments, were protein, total fat, $\mathrm{C} 16: 0$ saturated fatty acid (SFA) and $\mathrm{C} 18: 1 \mathrm{n} 9 \mathrm{c}$ monounsaturated fatty acid (MUFA). Moisture losses due to cooking resulted in an increase in the protein and cholesterol concentrations of the cooked cuts. Iron content was lower in the cooked loin cut but increased in the cooked leg cut when compared to the corresponding raw cuts. The vitamin B content of all three cooked lamb cuts was lower, although not significantly so, than that of the corresponding raw cuts. Lamb is a good source of protein, iron and the B vitamins, especially vitamin $\mathrm{B}_{12}$ when cooked.
\end{abstract}

\section{Article Outline}

1. Introduction

2. Materials and methods 


\subsection{Sampling}

\subsection{Sample preparation}

2.2.1. Right sides

2.2.2. Left sides

2.3. Preparation of the raw and cooked composite samples

2.4. Analyses

2.5. Statistical analyses

3. Results and discussion

3.1. Raw vs. cooked lamb

3.2. Raw cuts

3.3. Cooked cuts

3.4. Raw vs. cooked cuts

3.5. Recommended dietary allowances

3.6. Contribution of $100 \mathrm{~g}$ of cooked lamb to the nutrient requirements of males

3.7. Nutrient density

4. Conclusions

Acknowledgements

References

A knowledge of the chemical composition of foods is the first essential in dietary treatment of disease or in any quantitative study of human nutrition (McCance and Widdowson, 1940).

\section{Introduction}

As in many other countries, South Africa is actively involved in analysing foods for the compilation of food composition data. Currently, only $41 \%$ of all South African food values in the Medical Research Council's (MRC) tables are derived from South African foodstuffs (South African Food Composition Data (SAFCoD), 2000). The current data on mutton (lamb and sheep) that appears in the MRC's food composition tables of 1999, are derived from the United States Department of Agriculture (USDA) database (Sayed et al., 
1998). These data are not directly applicable and therefore it is appropriate that South Africa compiles sound scientific nutrient data for South African lamb and mutton. It is important that different countries have their own food composition tables for meat cuts (and products) such as lamb, mutton, beef and pork. The reason being, that different techniques are used to cut carcasses into primal cuts. Different age groups and fat codes of the animals in various countries make the interpretation of the results difficult (Schönfeldt, 1998) as the composition of the carcasses has a direct influence on the nutrient content thereof. The difference in climate, soil content and water composition of the various regions furthermore affects the nutrient content (specifically the minerals and vitamins) of the animal feed, as well as the production of vitamin $\mathrm{D}$ in the meat itself (Greenfield and Southgate, 2003). Table 1 provides a comparison and variation of macroand micro-nutrients when different databases are used, and illustrates that the use of different food composition tables may cause conflicting interpretation of dietary intake data. For instance, the iron content suggests that South African lamb is, on average slaughtered at a younger age than that of the United States of America's and British values and substantially younger than that of the Australian and New Zealand's. Iron content of meat is positively correlated with the age of the animal (Lawrie, 1998). 
Table 1.

Nutrient values in lean lamb, expressed per $100 \mathrm{~g}$ cooked edible portion, for selected countries

\begin{tabular}{|c|c|c|c|c|c|c|}
\hline Nutrients & Unit & South Africa ${ }^{a}$ & $\mathbf{U S A}^{\mathbf{b}}$ & $\mathbf{U K}^{\mathrm{c}}$ & Australiad $^{d}$ & New Zealand ${ }^{\mathrm{e}}$ \\
\hline & & $\begin{array}{l}\text { Cooked leg and } \\
\text { shank }\end{array}$ & $\begin{array}{l}\text { Cooked leg roasted, } \\
\text { lean and fat }\end{array}$ & $\begin{array}{l}\text { Cooked lamb } \\
\text { roast }\end{array}$ & $\begin{array}{l}\text { Cooked fresh leg and } \\
\text { shank half }\end{array}$ & $\begin{array}{l}\text { Cooked leg (shank } \\
\text { and sirloin) }\end{array}$ \\
\hline & & $\begin{array}{l}\text { Lean }( \pm 7 \% \\
\text { SCF) }\end{array}$ & Lean and fat & $90 \%$ meat & Trimmed to ${ }^{i_{n}}$ fat & $12 \%$ separable fat \\
\hline & & $100 \mathrm{~g}$ & $3 \mathrm{oz} / 85 \mathrm{~g}$ & $100 \mathrm{~g}$ & $100 \mathrm{~g}$ & $100 \mathrm{~g}$ \\
\hline \multicolumn{7}{|l|}{ Proximate analysis } \\
\hline Moisture & $\mathrm{g}$ & 66.03 & 57 & 58.70 & 59.2 & 63.92 \\
\hline Protein $(N \times 6.25)$ & $\mathrm{g}$ & 24.46 & 22 & 24.30 & 29.3 & 27.68 \\
\hline Fat & $\mathrm{g}$ & 7.67 & 14 & 13.30 & 11.9 & 7.01 \\
\hline Ash & $\mathrm{g}$ & 1.05 & - & - & 1.10 & 1.52 \\
\hline $\begin{array}{l}\text { Food energy } \\
\text { (calculated) }\end{array}$ & $\mathrm{kJ}$ & 722 & 931 & 905 & 937 & 757 \\
\hline \multicolumn{7}{|l|}{ Minerals } \\
\hline Magnesium (Mg) & $\mathrm{mg}$ & 23 & - & 24 & 19 & 21 \\
\hline Potassium (K) & $\mathrm{mg}$ & 303 & 266 & 350 & 290 & 183 \\
\hline Sodium (Na) & $\mathrm{mg}$ & 62 & 56 & 61 & 66 & 45 \\
\hline Zinc (Zn) & $\mathrm{mg}$ & 1.71 & - & 4.5 & 4.5 & 4.04 \\
\hline Iron $(\mathrm{Fe})$ & $\mathrm{mg}$ & 1.20 & 1.7 & 1.9 & 2.4 & 2.24 \\
\hline
\end{tabular}




\begin{tabular}{|c|c|c|c|c|c|c|}
\hline Nutrients & Unit & South Africa ${ }^{a}$ & $\mathbf{U S A}^{\mathbf{b}}$ & $\mathbf{U K}^{\mathrm{c}}$ & Australiad $^{d}$ & New Zealand ${ }^{\mathrm{e}}$ \\
\hline & & $\begin{array}{l}\text { Cooked leg and } \\
\text { shank }\end{array}$ & $\begin{array}{l}\text { Cooked leg roasted, } \\
\text { lean and fat }\end{array}$ & $\begin{array}{l}\text { Cooked lamb } \\
\text { roast }\end{array}$ & $\begin{array}{l}\text { Cooked fresh leg and } \\
\text { shank half }\end{array}$ & $\begin{array}{l}\text { Cooked leg (shank } \\
\text { and sirloin) }\end{array}$ \\
\hline & & $\begin{array}{l}\text { Lean }( \pm 7 \% \\
\text { SCF })\end{array}$ & Lean and fat & $90 \%$ meat & Trimmed to $\operatorname{lin}_{\text {fat }}$ & $12 \%$ separable fat \\
\hline & & $100 \mathrm{~g}$ & $3 \mathrm{oz} / 85 \mathrm{~g}$ & $100 \mathrm{~g}$ & $100 \mathrm{~g}$ & $100 \mathrm{~g}$ \\
\hline \multicolumn{7}{|l|}{ Vitamins } \\
\hline Thiamin $\left(\mathrm{B}_{1}\right)$ & $\mathrm{mg}$ & 0.06 & - & 0.15 & 0.06 & 0.12 \\
\hline Riboflavin $\left(\mathrm{B}_{2}\right)$ & $\mathrm{mg}$ & 0.05 & 0.23 & 0.27 & 0.25 & 0.50 \\
\hline $\operatorname{Niacin}\left(\mathrm{B}_{3}\right)$ & $\mathrm{mg}$ & 1.63 & 5.6 & 4.5 & 4.5 & 7.51 \\
\hline Pyridoxine $\left(\mathrm{B}_{6}\right)$ & $\mathrm{mg}$ & 0.10 & - & 0.22 & - & 0.14 \\
\hline $\begin{array}{l}\text { Cyanocobalamin } \\
\left(\mathrm{B}_{12}\right)\end{array}$ & $\mu g$ & 1.06 & - & 4 & - & 2.63 \\
\hline \multicolumn{7}{|l|}{ Lipids } \\
\hline SFA & $\mathrm{g}$ & 4.07 & 5.9 & 6.1 & 6.1 & 3.05 \\
\hline MUFA & $\mathrm{g}$ & 3.16 & 5.9 & 5.3 & 4.3 & 2.75 \\
\hline PUFA & $\mathrm{g}$ & 0.29 & 1.0 & 0.7 & 0.2 & 0.41 \\
\hline Cholesterol & $\mathrm{mg}$ & 92 & 79 & 98 & 109 & 10063 \\
\hline
\end{tabular}

SCF: subcutaneous fat; SFA: saturated fatty acids; MUFA: monounsaturated fatty acids; PUFA: polyunsaturated fatty acids; :unreliable values omitted, currently being re-analysed.

${ }^{a}$ Data from this study.

${ }^{\mathrm{b}}$ Gebhardt and Thomas (2002). 
${ }^{\mathrm{c}}$ Chan et al. (1996).

${ }^{\mathrm{d}}$ Lewis et al. (1995, vol. 1).

${ }^{\mathrm{e}}$ United Stated Department of Agriculture (1989).

Another aspect of nutrition is food choices, which have a direct impact on a person's health status. These choices are repeated over a long period (years or decades) and have major positive or negative health effects (Kruger et al., 2003). Therefore, in order to evaluate a person's food intake, according to the Recommended Dietary Allowance (RDA), the nutritional composition of the diet is calculated by making use of food composition databases, either in computerised software or printed form. This information provides the average nutrient content for a given amount of food, based on the chemical analysis of a number of food samples. Information on the nutrient content of a particular food can also be used as a standard reference to determine nutrients obtained from the foodstuff consumed throughout a country (Latham, 1997).

In developing countries, such as in South Africa, one of the major health concerns is the poor nutrient content, specifically micronutrients, of the diet. Iron deficiency resulting in anaemia, is the most common deficiency in the world that causes ill health. Data from the South African National Food Consumption Survey (Steyn et al., 2000) showed that children aged 1-9 years had low mean intakes of iron, protein, B-vitamins and minerals such as zinc and iodine. Consequently, nutrient deficiencies are widespread in all societies in South Africa.

Certain segments of our population are consuming significant amounts of lamb, and yet there is a lack of comprehensive, South African nutrient composition data on lamb. Following the need for data on the composition of South African lamb and mutton, as identified by the SAFCoD, the Animal Nutrition and Animal Products Institute of the Agricultural Research Council, Irene initiated a research focus area in 1988 to determine the nutrient content of South African animal products. Therefore, the aim of this research was to determine the nutrient composition of South African lamb in order to incorporate the data into the Food Composition Tables of the Medical Research Council of South Africa. 


\section{Materials and methods}

\subsection{Sampling}

The South African classification system (National Department of Agriculture, 1990, p. 914 ) is designed to describe carcasses according to tissue composition. To this end, age is described according to the number of permanent incisors with age class $A=0$ teeth, $\mathrm{AB}=1-2$ teeth, $\mathrm{B}=3-6$ teeth and $\mathrm{C}=$ more than 6 teeth, while carcasses are grouped into 6 fat classes by means of visual appraisal (fatness class from $0=$ no fat to $6=$ excessively over fat). According to the fatness classification (National Department of Agriculture, 1990) an A2 lamb can have at least $1 \mathrm{~mm}$, but not more than $4 \mathrm{~mm}$ fat, and more than $5.6 \%$ but not more than $8.5 \%$ subcutaneous fat (SCF) (average of $7 \% \mathrm{SCF}$ ).

Three wholesale cuts (leg, loin, shoulder) of South African lamb (Dorper and Merino) of an A-age group ( 0 incisors) with a fat code of 2 (lean $=1-4 \mathrm{~mm}$ fat with $\pm 7 \% \mathrm{SCF}$ ) were selected. The nutrient content of meat and fat of each of the three wholesale cuts of one side (right) were analysed for raw nutrient values and three wholesale cuts of one side (left) were analysed for cooked nutrient values.

The A2 class lamb carcasses [(lean animals ( $\pm 7 \%$ SCF) with no incisors)] were selected in co-operation with the South African Meat Industry Company (SAMIC) and the Red Meat Producers Organization (RPO). Market share was used as the main factor in the selection of the two breeds. The attributes used were selected based on the fact that sheep are drawn from main production areas, supplying the main abattoirs. The meat samples, incorporated in the study, comprised of the most commonly consumed carcasses in South Africa (Japie van der Westhuizen, personal communication, 2003), namely Dorper $(n=9)$ and Merino $(n=9)$ carcasses of the A age group with a fatness level of $2( \pm 7 \% \mathrm{SCF})$. Sheep slaughtered at these abattoirs are drawn from the three main production areas in South Africa namely the Karoo, Kalahari and Ermelo districts. The carcasses are classified according to the South African classification system at the abattoirs. The lambs were slaughtered using standard commercial procedures during four consecutive weeks. After selection the carcasses were transported in a refrigerated truck $\left(4-6{ }^{\circ} \mathrm{C}\right)$ to the Meat Industry Centre of the ARC-ANPI, Irene. Upon arrival all the carcasses were weighed, covered with plastic wrap to prevent moisture loss and chilled at $4{ }^{\circ} \mathrm{C}$ overnight and dissected the following day. The lamb carcasses consisted of the skinned, eviscerated 
body from which the head and feet were removed. Carcasses were sectioned down the vertebral column with a band saw and then subdivided into the following primal cuts: neck, thick rib, flank, shoulder, breast, rib, loin, chump, leg and shank. The right sides of the carcasses were used to determine the physical cut composition as well as by calculation for the whole carcass (these data will be presented in another article). The raw nutrient contents for some macro- and micronutrients for each cut were also determined. Three cuts, representing the most commonly consumed cuts, (shoulder, loin and leg) from the left sides were used to determine the cooked nutrient composition thereof. These cuts were vacuum packed and frozen at $-20^{\circ} \mathrm{C}$ until the cooking process commenced. The raw and cooked nutrient data of the three cuts was compared. The assumption, as confirmed by Kirton et al. (1962), is that the composition of the two sides is similar.

\subsection{Sample preparation}

\subsubsection{Right sides}

A trained deboning team was responsible for the physical dissection of the raw lamb carcasses that were weighed prior to being divided into primal cuts. The cuts from the right sides were dissected by knife into three portions namely meat [muscle+intramuscular fat (i.e. fat within the muscle)], bone and subcutaneous fat, in an environmentally controlled $\left(10^{\circ} \mathrm{C}\right)$ abattoir.

\subsubsection{Left sides}

The shoulder, loin and leg cuts were thawed, weighed and cooked according to standardised cooking methods (leg, combination dry and moist heat cooking method; loin, dry heat cooking method; shoulder, moist heat cooking method) in identical Mielé ovens at $160{ }^{\circ} \mathrm{C}$, to an internal temperature of $71{ }^{\circ} \mathrm{C}$, measured in the geometrical centre of the cut (American Meat Science Association, 1995). It was then dissected into three portions namely meat (muscle+intramuscular fat), bone and subcutaneous fat, in an environmentally controlled $\left(10^{\circ} \mathrm{C}\right)$ abattoir by the team of experienced dissectors. 


\subsection{Preparation of the raw and cooked composite samples}

In order to comply with the new Draft Regulations relating to the Labeling and Advertising of Foodstuffs, as part of the Foodstuffs, Cosmetics and Disinfectants Act (1972, http://0-www.doh.gov.za.innopac.up.ac.za:80/department/dir_foodcontr.html), it was proposed that a composite of three carcasses would be used as a basis of the study. The use of composite samples for analysis rather than individual samples is justified by budgeting constraints and is an accepted approach in food composition studies (Greenfield and Southgate, 2003). Three wholesale cuts (leg, loin, shoulder) were selected. The meat and fat of each of the three wholesale cuts of one side (right) were analysed for raw and three wholesale cuts of one side (left) were analysed for cooked nutrients (proximate analysis, vitamins, minerals, fatty acids and cholesterol). To prepare the composite samples, the meat and fat, respectively, of all the replications of each raw and cooked cuts were combined and cubed, thoroughly mixed and then minced, firstly through a $5 \mathrm{~mm}$ and then through a $3 \mathrm{~mm}$ mesh plate. Samples of $300 \mathrm{~g}$ meat and separable fat were homogenised with an Ultra Turrax T25 homogeniser after mincing to ensure a proper homogenised sample. These samples were then either placed in glass bottles prior to being frozen, or into aluminium trays covered with a vacuum bag prior to being freeze-dried.

\subsection{Analyses}

All the analytical procedures (Table 2) for the nutrient content of the lamb samples were done on a double blind basis in the laboratories (ISO/IEC 17025:2005) that form part of the South African National Accreditation Services (SANAS). Control samples form part of the daily routine in these laboratories to assure the quality of results. On the operational level, all sampling were representative and handled with utmost accuracy. A proper sampling plan was followed with representative samples from each area and sufficient replications of each sample were used to ensure statistically reliable and valid data. 
Table 2.

Methods used for the nutrient analyses of raw and cooked South African lamb

\begin{tabular}{|l|l|}
\hline Analyses & Method \\
\hline Water & Association of Official Analytical Chemists (2005) method 950.46 \\
\hline Ash & Association of Official Analytical Chemists (2005) method 920.153 \\
\hline Protein & International Dairy Foundation Standard 20B (1993) \\
\hline Fat & International Dairy Foundation Standard 20B (1996) \\
\hline Energy & Bomb calorimeter to calculate \\
\hline Minerals & Ion chromatography (Dionex System 20001, 1988) \\
\hline $\begin{array}{l}\text { Water-soluble } \\
\text { vitamins }\end{array}$ & High performance liquid chromatography (Fellman et al., 1992) \\
\hline Fatty acid profile & Gas chromatography (Christopherson and Glass, 1969) \\
\hline Cholesterol & Gas chromatography (Smuts et al., 1992) \\
\hline
\end{tabular}

\subsection{Statistical analyses}

Nutrient data obtained from the analyses were entered on a spreadsheet using Microsoft Excel (2000). Data was statistically analysed by the ARC-Biometry Unit using GenStat for Windows (2003). The significance of all the variables measured for each sample was tested with split-plot analysis of variance (ANOVA), whereby the main effect of the cuts (whole plots) and treatment (raw and cooked sub-plots), as well as the cut-by-treatment interaction was tested at the $5 \%$ level of significance $(p \leqslant 0.05)$. If a main effect was significant, the Fishers' protected $t$-test with Least Significant Difference (LSD) was applied, to determine the direction of the differences between mean values (Snedecor and Cochran, 1980).

\section{Results and discussion}

The majority of the population in most developing countries consumes meat (and meat products) that significantly contributes to nutrient intake for most individuals. Meat provides the following nutrients, namely, fats which provide energy and contain essential 
fatty acids, minerals such as iron and zinc in a readily-digestible form, vitamins, particularly vitamin $\mathrm{B}_{12}$ and a range of amino acids essential for growth and development in the human body (Enser, 2000).

\subsection{Raw vs. cooked lamb}

The nutrient values of cooked lamb are more useful to the consumers than raw values. The raw values (Table 3 ) provide baseline information that could be used to evaluate production and marketing effects on nutrient composition. Cooked values, on the other hand, provide information on what is consumed. The differences in the amount of nutrients between raw and cooked meat cuts can be used to calculate nutrient retention. The mean values and standard deviations of this study for proximate analysis, minerals, certain water-soluble vitamins, cholesterol and fatty acids in a composite of raw and cooked meat (100 g edible portion) from three retail cuts of South African lamb (A2, $\pm 7 \% \mathrm{SCF}$ ), are presented in Table 3 , Table 4 and Table 5.

Table 3.

Mean values of the nutrient composition for raw and cooked, expressed $100 \mathrm{~g}$ edible portion of lean lamb $( \pm 7 \% \mathrm{SCF})^{\mathrm{a}}$

\begin{tabular}{|c|c|c|c|c|c|}
\hline \multicolumn{6}{|c|}{100 g edible portion of lean lamb $( \pm 7 \%$ SCF $)(n=18)$} \\
\hline Nutrients analysed & Unit & $p$-value & $\mathbf{S E M}^{\mathbf{b}}$ & Raw & Cooked \\
\hline \multicolumn{6}{|l|}{ Proximate analysis } \\
\hline Moisture & $\mathrm{g}$ & $<0.001$ & 0.395 & 71.5 & 65.4 \\
\hline Protein $(N \times 6.25)$ & $\mathrm{g}$ & $<0.001$ & 0.406 & 18.3 & 25.1 \\
\hline Fat & $\mathrm{g}$ & 0.217 & 0.306 & 9.01 & 8.44 \\
\hline Ash & $\mathrm{g}$ & $<0.001$ & 0.287 & 2.88 & 1.07 \\
\hline Food energy (calculated) & $\mathrm{kJ}$ & $<0.001$ & 14.4 & 644 & 745 \\
\hline \multicolumn{6}{|l|}{ Minerals } \\
\hline Magnesium (Mg) & $\mathrm{mg}$ & 0.405 & 1.261 & 20.1 & 21.7 \\
\hline Potassium (K) & $\mathrm{mg}$ & 0.852 & 62.6 & 291 & 298 \\
\hline
\end{tabular}




\begin{tabular}{|c|c|c|c|c|c|}
\hline \multicolumn{6}{|c|}{100 g edible portion of lean lamb $( \pm 7 \%$ SCF $)(n=18)$} \\
\hline Nutrients analysed & Unit & $p$-value & $\mathbf{S E M}^{\mathbf{b}}$ & Raw & Cooked \\
\hline Sodium $(\mathrm{Na})$ & $\mathrm{mg}$ & 0.302 & 7.90 & 83.4 & 71.3 \\
\hline Zinc $(\mathrm{Zn})$ & $\mathrm{mg}$ & 0.196 & 0.275 & 2.25 & 1.72 \\
\hline Iron $(\mathrm{Fe})$ & $\mathrm{mg}$ & 0.260 & 0.198 & 0.96 & 0.63 \\
\hline \multicolumn{6}{|l|}{ Vitamins } \\
\hline Thiamin $\left(\mathrm{B}_{1}\right)$ & $\operatorname{mg}$ & 0.017 & 0.015 & 0.10 & 0.04 \\
\hline Riboflavin $\left(\mathrm{B}_{2}\right)$ & $\mathrm{mg}$ & 0.102 & 0.013 & 0.09 & 0.05 \\
\hline Niacin $\left(B_{3}\right)$ & $\mathrm{mg}$ & 0.869 & 0.248 & 1.47 & 1.42 \\
\hline Pyridoxine $\left(\mathrm{B}_{6}\right)$ & $\mathrm{mg}$ & 0.003 & 0.054 & 0.40 & 0.12 \\
\hline Cyanocobalamin $\left(\mathrm{B}_{12}\right)$ & $\mu \mathrm{g}$ & 0.003 & 0.493 & 3.54 & 0.93 \\
\hline \multicolumn{6}{|l|}{ Lipids $^{\underline{\mathrm{c}}}$} \\
\hline \multicolumn{6}{|l|}{ Saturated fatty acids } \\
\hline $14: 0$ & $\mathrm{~g}$ & 0.297 & 0.038 & 0.57 & 0.50 \\
\hline $16: 0$ & $\mathrm{~g}$ & 0.111 & 0.086 & 2.22 & 1.99 \\
\hline $18: 0$ & $\mathrm{~g}$ & 0.002 & 0.048 & 1.46 & 1.07 \\
\hline $20: 0$ & $\mathrm{~g}$ & 0.137 & 0.002 & 0.02 & 0.02 \\
\hline \multicolumn{6}{|c|}{ Monounsaturated fatty acids } \\
\hline $16: 1$ & $\mathrm{~g}$ & 0.983 & 0.009 & 0.19 & 0.19 \\
\hline $18: \ln 9 t$ & $\mathrm{~g}$ & 0.009 & 0.017 & 0.31 & 0.21 \\
\hline $18: \ln 9 \mathrm{c}$ & $\mathrm{g}$ & 0.001 & 0.050 & 3.12 & 2.65 \\
\hline \multicolumn{6}{|c|}{ Polyunsaturated fatty acids } \\
\hline $18: 2 \mathrm{n} 6 \mathrm{t}$ & $\mathrm{g}$ & 0.215 & 0.003 & 0.02 & 0.02 \\
\hline $18: 2 \mathrm{n} 6 \mathrm{c}$ & $\mathrm{g}$ & 0.233 & 0.014 & 0.25 & 0.22 \\
\hline Cholesterol & $\mathrm{mg}$ & 0.001 & 4.15 & 62.8 & 87.7 \\
\hline
\end{tabular}

p-value: F-probability to test for significant differences between composite samples (cuts). 
The significance of all the variables measured for each sample was tested with split-plot analysis of variance (ANOVA), whereby the main effect of the cuts ( $n=12$ whole [plots) and treatment ( $n=18$ raw and cooked sub-plots), as well as the cut-by-treatment interaction $(n=6)$ was tested for the $5 \%$ level of significance $(p \leqslant 0.05)$.

${ }^{a}$ Except for B vitamins, the modes of expression used are according to Greenfield and Southgate (2003).

${ }^{\mathrm{b}}$ SEM: standard error of means.

${ }^{\mathrm{c}}$ Lipids: fatty acids: represent total fatty acids including free fatty acids.

Table 4.

Mean values of the nutrient composition of three raw and three cooked cut, expressed per $100 \mathrm{~g}$ edible portion of lean lamb $( \pm 7 \% \mathrm{SCF})^{\mathrm{a}}$

\begin{tabular}{|c|c|c|c|c|c|c|c|c|c|c|c|}
\hline \multirow[t]{2}{*}{$\begin{array}{l}\text { Nutrients } \\
\text { analysed }\end{array}$} & \multirow[t]{2}{*}{ Unit } & \multicolumn{5}{|c|}{ Raw cuts ( $n=12)$} & \multicolumn{5}{|c|}{ Cooked cuts $(n=12)$} \\
\hline & & $\begin{array}{l}p \text { - } \\
\text { value }\end{array}$ & $\mathbf{S E M}^{\mathbf{b}}$ & Shoulder & Loin & Leg & $\begin{array}{l}p \text { - } \\
\text { value }\end{array}$ & $\mathbf{S E M}^{\mathbf{b}}$ & Shoulder & Loin & Leg \\
\hline \multicolumn{12}{|c|}{ Proximate analysis } \\
\hline Moisture & g & 0.012 & 0.741 & $70.8^{\mathrm{a}}$ & $70.1^{\mathrm{a}}$ & $73.7^{\mathrm{b}}$ & $<0.001$ & 0.478 & $66.8^{\mathrm{b}}$ & $63.5^{\mathrm{a}}$ & $66.0^{\mathrm{b}}$ \\
\hline $\begin{array}{l}\text { Protein } \\
(N \times 6.25)\end{array}$ & $\mathrm{g}$ & 0.346 & 0.638 & 18.0 & 17.8 & 19.0 & $<0.001$ & 0.541 & $23.1^{\mathrm{a}}$ & $27.8^{\mathrm{b}}$ & $24.5^{\mathrm{a}}$ \\
\hline Fat & $\mathrm{g}$ & 0.003 & 0.814 & $9.63^{\mathrm{a}}$ & $11.3^{\mathrm{a}}$ & $6.15^{\mathrm{b}}$ & 0.018 & 0.515 & $9.86^{\mathrm{a}}$ & $7.80^{\mathrm{b}}$ & $7.67^{\mathrm{b}}$ \\
\hline Ash & $\mathrm{g}$ & 0.918 & 0.711 & 2.93 & 2.65 & 3.06 & $<0.001$ & 0.016 & $0.95^{\mathrm{a}}$ & $1.20^{\mathrm{c}}$ & $1.05^{\mathrm{b}}$ \\
\hline $\begin{array}{l}\text { Food energy } \\
\text { (calculated) }\end{array}$ & $\mathrm{kJ}$ & 0.014 & 33.9 & $662.0^{\mathrm{a}}$ & $718^{\mathrm{a}}$ & $552^{\mathrm{b}}$ & 0.438 & 20.4 & 757 & 755 & 722 \\
\hline \multicolumn{12}{|l|}{ Minerals } \\
\hline $\begin{array}{l}\text { Magnesium } \\
(\mathrm{Mg})\end{array}$ & $\mathrm{mg}$ & 0.076 & 3.01 & 13.9 & 22.7 & 23.8 & $<0.001$ & 0.674 & $17.7^{\mathrm{a}}$ & $24.2^{\mathrm{b}}$ & $22.9^{b}$ \\
\hline Potassium (k) & $\mathrm{mg}$ & 0.134 & 51.7 & 201. & 323 & 351 & $<0.001$ & 7.51 & $261^{\mathrm{a}}$ & $331^{\mathrm{c}}$ & $303^{\mathrm{b}}$ \\
\hline Sodium $(\mathrm{Na})$ & $\mathrm{mg}$ & 0.495 & 19.1 & 68.0 & 101 & 82 & $<0.001$ & 1.528 & $68.9^{\mathrm{b}}$ & $83.3^{c}$ & $61.9^{\mathrm{a}}$ \\
\hline Zinc (Zn) & $\mathrm{mg}$ & 0.512 & 0.474 & 1.99 & 2.05 & 2.71 & 0.208 & 0.357 & 1.24 & 2.20 & 1.71 \\
\hline
\end{tabular}




\begin{tabular}{|c|c|c|c|c|c|c|c|c|c|c|c|}
\hline \multicolumn{12}{|c|}{100 g edible portion of raw and cooked lean lamb $( \pm 7 \%$ SCF) } \\
\hline \multirow[t]{2}{*}{$\begin{array}{l}\text { Nutrients } \\
\text { analysed }\end{array}$} & \multirow[t]{2}{*}{ Unit } & \multicolumn{5}{|c|}{ Raw cuts $(n=12)$} & \multicolumn{5}{|c|}{ Cooked cuts $(n=12)$} \\
\hline & & $\begin{array}{l}p \text { - } \\
\text { value }\end{array}$ & $\mathbf{S E M}^{\mathbf{b}}$ & Shoulder & Loin & Leg & $\begin{array}{l}p \text { - } \\
\text { value }\end{array}$ & $\mathbf{S E M}^{\mathbf{b}}$ & Shoulder & Loin & Leg \\
\hline Iron $(\mathrm{Fe})$ & $\mathrm{mg}$ & 0.397 & 0.197 & 0.75 & 0.99 & 1.14 & 0.227 & 0.439 & - & 0.62 & 1.20 \\
\hline \multicolumn{12}{|l|}{ Vitamins } \\
\hline Thiamin $\left(\mathrm{B}_{1}\right)$ & $\mathrm{mg}$ & 0.822 & 0.021 & 0.11 & 0.09 & 0.10 & 0.740 & 0.030 & 0.03 & 0.04 & 0.06 \\
\hline Riboflavin $\left(\mathrm{B}_{2}\right)$ & $\mathrm{mg}$ & 0.954 & 0.027 & 0.09 & 0.08 & 0.09 & 0.535 & 0.018 & 0.04 & 0.07 & 0.05 \\
\hline $\operatorname{Niacin}\left(B_{3}\right)$ & $\mathrm{mg}$ & 0.525 & 0.479 & 1.70 & 1.02 & 1.71 & 0.736 & 0.342 & 1.37 & 1.25 & 1.63 \\
\hline Pyridoxine $\left(\mathrm{B}_{6}\right)$ & $\mathrm{mg}$ & 0.099 & 0.122 & 0.26 & 0.32 & 0.64 & 0.716 & 0.030 & 0.12 & 0.13 & 0.10 \\
\hline $\begin{array}{l}\text { Cyanocobalamin } \\
\left(\mathrm{B}_{12}\right)\end{array}$ & $\mu \mathrm{g}$ & 0.803 & 1.085 & 4.02 & 3.61 & 3.00 & 0.697 & 0.188 & 0.8 & 0.2 & 1.1 \\
\hline
\end{tabular}

\section{Lipids $^{\mathrm{c}}$}

\section{Saturated fatty acids}

\begin{tabular}{|l|l|l|l|l|l|l|l|l|l|l|l|}
\hline $14: 0$ & $\mathrm{~g}$ & 0.111 & 0.095 & 0.62 & 0.69 & 0.39 & 0.694 & 0.066 & 0.50 & 0.47 & 0.42 \\
\hline $16: 0$ & $\mathrm{~g}$ & 0.010 & 0.238 & $2.50^{\mathrm{a}}$ & $2.67^{\mathrm{a}}$ & $1.50^{\mathrm{b}}$ & 0.562 & 0.120 & 2.06 & 1.97 & 1.86 \\
\hline $18: 0$ & $\mathrm{~g}$ & $<0.001$ & 0.132 & $1.64^{\mathrm{a}}$ & $1.86^{\mathrm{a}}$ & $0.88^{\mathrm{b}}$ & 0.329 & 0.101 & 1.10 & 1.08 & 1.30 \\
\hline $20: 0$ & $\mathrm{~g}$ & 0.120 & 0.006 & 0.03 & 0.03 & 0.01 & 0.045 & 0.005 & 0.02 & 0.01 & 0.02 \\
\hline
\end{tabular}

Monounsaturated fatty acids

\begin{tabular}{|l|l|l|l|l|l|l|l|l|l|l|l|}
\hline $16: 1$ & $\mathrm{~g}$ & 0.152 & 0.028 & 0.21 & 0.21 & 0.14 & 0.113 & 0.009 & 0.20 & 0.18 & 0.16 \\
\hline $18: 1 \mathrm{n} 9 \mathrm{t}$ & $\mathrm{g}$ & 0.061 & 0.061 & 0.33 & 0.42 & 0.19 & 0.907 & 0.048 & 0.23 & 0.24 & 0.21 \\
\hline $18: 1 \mathrm{ln} 9 \mathrm{c}$ & $\mathrm{g}$ & $<0.001$ & 0.206 & $3.53^{\mathrm{a}}$ & $3.70^{\mathrm{a}}$ & $2.14^{\mathrm{b}}$ & 0.294 & 0.102 & 2.88 & 2.63 & 2.71 \\
\hline
\end{tabular}

Polyunsaturated fatty acids

\begin{tabular}{|l|l|l|l|l|l|l|l|l|l|l|l|}
\hline $18: 2 \mathrm{n} 6 \mathrm{t}$ & $\mathrm{G}$ & 0.607 & 0.005 & 0.03 & 0.02 & 0.02 & 0.886 & 0.005 & 0.02 & 0.02 & 0.02 \\
\hline $18: 2 \mathrm{n} 6 \mathrm{c}$ & $\mathrm{G}$ & 0.018 & 0.029 & $0.28^{\mathrm{a}}$ & $0.29^{\mathrm{a}}$ & $0.17^{\mathrm{b}}$ & 0.891 & 0.034 & 0.24 & 0.22 & 0.24 \\
\hline Cholesterol & $\mathrm{Mg}$ & 0.966 & 6.09 & 64.0 & 61.8 & 62.7 & 0.732 & 6.23 & 85.0 & 86.3 & 91.7 \\
\hline
\end{tabular}

— Unreliable values omitted, currently being re-analysed.

p-value: F-probability to test for significant differences between composite samples. 
The significance of all the variables measured for each sample was tested with split-plot analysis of variance (ANOVA), whereby the main effect of the cuts ( $n=12$ whole plots) and treatment ( $n=18$ raw and cooked sub-plots), as well as the cut-by-treatment interaction $(n=6)$ was tested at the $5 \%$ level of significance ( $p \leqslant 0.05)$. Means with different letters $(\mathrm{a}, \mathrm{b}$ or $\mathrm{c})$ are significant different.

${ }^{a}$ Except for B vitamins, the modes of expression used are according to Greenfield and Southgate (2003).

${ }^{\mathrm{b}}$ SEM: standard error of means.

${ }^{\mathrm{c}}$ Lipids: fatty acids: represent total fatty acids including free fatty acids.

Table 5.

Mean values of the nutrient composition for the interaction between raw and cooked cut, expressed per $100 \mathrm{~g}$ edible portion of lean lamb $( \pm 7 \% \mathrm{SCF})^{\mathrm{a}}$

\begin{tabular}{|c|c|c|c|c|c|c|c|c|c|}
\hline \multirow[t]{3}{*}{ Nutrients analysed } & \multirow[t]{3}{*}{ Unit } & \multirow[t]{3}{*}{$\begin{array}{l}p \text { - } \\
\text { value }\end{array}$} & \multirow[t]{3}{*}{ SEM $^{\mathbf{b}}$} & \multicolumn{6}{|c|}{100 g edible portion of LEAN Lamb ( $\pm 7 \%$ SCF) } \\
\hline & & & & \multicolumn{3}{|l|}{ Raw $(n=6)$} & \multicolumn{3}{|c|}{ Cooked $(n=6)$} \\
\hline & & & & Shoulder & Loin & Leg & Shoulder & Loin & Leg \\
\hline \multicolumn{10}{|l|}{ Proximate analysis } \\
\hline Moisture & $\mathrm{g}$ & 0.054 & 0.624 & 70.8 & 70.1 & 73.7 & 66.8 & 63.5 & 66.0 \\
\hline Protein $(\mathrm{N} \times 6.25)$ & $\mathrm{g}$ & 0.007 & 0.591 & $18.0^{\mathrm{a}}$ & $17.8^{\mathrm{a}}$ & $19.0^{\mathrm{a}}$ & $23.1^{b}$ & $27.8^{\mathrm{c}}$ & $24.5^{\mathrm{b}}$ \\
\hline Fat & $\mathrm{g}$ & 0.007 & 0.681 & $9.63^{b}$ & $11.25^{\mathrm{b}}$ & $6.15^{\mathrm{a}}$ & $9.86^{\mathrm{b}}$ & $7.80^{\mathrm{a}}$ & $7.67^{\mathrm{a}}$ \\
\hline Ash & $\mathrm{g}$ & 0.824 & 0.503 & 2.93 & 2.65 & 3.06 & 0.95 & 1.19 & 1.05 \\
\hline $\begin{array}{l}\text { Food energy } \\
\text { (calculated) }\end{array}$ & $\mathrm{kJ}$ & 0.059 & 28.0 & 662 & 718 & 552 & 757 & 755 & 723 \\
\hline \multicolumn{10}{|l|}{ Minerals } \\
\hline Magnesium (Mg) & $\mathrm{mg}$ & 0.545 & 2.182 & 13.9 & 22.7 & 23.8 & 18.0 & 24.2 & 22.9 \\
\hline Potassium (K) & $\mathrm{mg}$ & 0.340 & 36.9 & 201 & 323 & 351 & 261 & 330 & 303 \\
\hline Sodium $(\mathrm{Na})$ & $\mathrm{mg}$ & 0.716 & 13.53 & 67.9 & 100.7 & 81.6 & 68.9 & 83.3 & 61.8 \\
\hline Zinc (Zn) & $\mathrm{mg}$ & 0.470 & 0.419 & 1.99 & 2.05 & 2.71 & 1.24 & 2.20 & 1.71 \\
\hline Iron $(\mathrm{Fe})$ & $\mathrm{mg}$ & 0.564 & 0.340 & 0.75 & 0.99 & 1.14 & - & 0.62 & 1.20 \\
\hline
\end{tabular}




\begin{tabular}{|c|c|c|c|c|c|c|c|c|c|}
\hline \multirow[t]{3}{*}{ Nutrients analysed } & \multirow[t]{3}{*}{ Unit } & \multirow[t]{3}{*}{$\begin{array}{l}p \text { - } \\
\text { value }\end{array}$} & \multirow[t]{3}{*}{$\mathbf{S E M}^{\mathbf{b}}$} & \multicolumn{6}{|c|}{100 g edible portion of LEAN Lamb $( \pm 7 \%$ SCF $)$} \\
\hline & & & & \multicolumn{3}{|c|}{ Raw $(n=6)$} & \multicolumn{3}{|c|}{ Cooked $(n=6)$} \\
\hline & & & & Shoulder & Loin & Leg & Shoulder & Loin & Leg \\
\hline \multicolumn{10}{|l|}{ Vitamins } \\
\hline Thiamin $\left(\mathrm{B}_{1}\right)$ & $\mathrm{mg}$ & 0.682 & 0.026 & 0.11 & 0.09 & 0.10 & 0.03 & 0.04 & 0.06 \\
\hline Riboflavin $\left(\mathrm{B}_{2}\right)$ & $\mathrm{mg}$ & 0.700 & 0.023 & 0.09 & 0.08 & 0.09 & 0.04 & 0.07 & 0.05 \\
\hline $\operatorname{Niacin}\left(B_{3}\right)$ & $\mathrm{mg}$ & 0.807 & 0.416 & 1.70 & 1.02 & 1.71 & 1.37 & 1.25 & 1.63 \\
\hline Pyridoxine $\left(\mathrm{B}_{6}\right)$ & $\mathrm{mg}$ & 0.101 & 0.089 & 0.26 & 0.32 & 0.64 & 0.12 & 0.13 & 0.10 \\
\hline Cyanocobalamin $\left(\mathrm{B}_{12}\right)$ & $\mu g$ & 0.768 & 0.779 & 4.02 & 3.61 & 3.00 & 0.83 & 0.91 & 1.05 \\
\hline \multicolumn{10}{|l|}{ Lipids $^{\mathrm{c}}$} \\
\hline \multicolumn{10}{|l|}{ Saturated fatty acids } \\
\hline $14: 0$ & $\mathrm{~g}$ & 0.093 & 0.097 & 0.62 & 0.69 & 0.39 & 0.59 & 0.42 & 0.50 \\
\hline $16: 0$ & g & 0.020 & 0.236 & $2.50^{\mathrm{b}}$ & $2.67^{b}$ & $1.50^{\mathrm{a}}$ & $2.05^{\mathrm{a}}$ & $1.92^{\mathrm{a}}$ & $2.00^{\mathrm{a}}$ \\
\hline $18: 0$ & g & 0.001 & 0.140 & $1.64^{\mathrm{b}}$ & $1.86^{\mathrm{b}}$ & $0.88^{\mathrm{a}}$ & $0.78^{\mathrm{a}}$ & $1.15^{\mathrm{a}}$ & $1.27^{\mathrm{b}}$ \\
\hline $20: 0$ & g & 0.034 & 0.006 & $0.03^{\mathrm{a}}$ & $0.03^{\mathrm{a}}$ & $0.01^{\mathrm{b}}$ & $0.01^{\mathrm{b}}$ & $0.02^{b}$ & $0.03^{\mathrm{a}}$ \\
\hline \multicolumn{10}{|c|}{ Monounsaturated fatty acids } \\
\hline $16: 1$ & g & 0.100 & 0.026 & 0.21 & 0.21 & 0.14 & 0.22 & 0.17 & 0.18 \\
\hline $18: \ln 9 \mathrm{t}$ & g & 0.076 & 0.057 & 0.33 & 0.42 & 0.19 & 0.22 & 0.01 & 0.18 \\
\hline $18: \ln 9 \mathrm{c}$ & g & $<0.001$ & 0.200 & $3.53^{\mathrm{c}}$ & $3.70^{\mathrm{c}}$ & $2.14^{\mathrm{a}}$ & $2.60^{\mathrm{b}}$ & $2.63^{\mathrm{b}}$ & $2.70^{\mathrm{b}}$ \\
\hline \multicolumn{10}{|c|}{ Polyunsaturated fatty acids } \\
\hline $18: 2 \mathrm{n} 6 \mathrm{t}$ & g & 0.567 & 0.005 & 0.03 & 0.02 & 0.02 & 0.02 & 0.02 & 0.02 \\
\hline $18: 2 \mathrm{n} 6 \mathrm{c}$ & $\mathrm{g}$ & 0.069 & 0.033 & 0.28 & 0.29 & 0.17 & 0.20 & 0.24 & 0.23 \\
\hline Cholesterol & $\mathrm{mg}$ & 0.857 & 6.26 & 64 & 62 & 63 & 85 & 86 & 92 \\
\hline
\end{tabular}

- Unreliable values omitted, currently being re-analysed.

p-value: F-probability to test for significant differences between samples.

The significance of all the variables measured for each sample was tested with split-plot analysis of variance (ANOVA), whereby the main effect of the cuts ( $n=12$ - whole plots) 
and treatment ( $n=18$ raw and cooked sub-plots), as well as the cut-by-treatment interaction ( $n=6)$ was tested at the $5 \%$ level of significance ( $p \leqslant 0.05$ ). Means with different letters ( $a, b$ or $c$ ) are significantly different.

${ }^{a}$ Except for B vitamins, the modes of expression used are according to Greenfield and Southgate (2003).

${ }^{\mathrm{b}}$ SEM: standard error of means.

${ }^{\mathrm{c}}$ Lipids: fatty acids: represent total fatty acids including free fatty acids.

\subsection{Raw cuts}

The nutrient components exhibiting the greatest differences between the three raw cuts, were moisture, total fat, energy, $\mathrm{C} 16: 0$ and $\mathrm{C} 18: 0$ saturated fatty acids, $\mathrm{C} 18: \ln 9 \mathrm{c}$ monounsaturated fatty acid and C18:2n6c polyunsaturated fatty acid. According to the results presented in Table 4, the leg cut differed significantly from the shoulder and loin cuts for moisture, fat and energy. The moisture content of the leg cut was significantly higher than the shoulder and the loin. Of the three cuts, the leg had significantly less fat when compared to the shoulder and the loin cut which contained the highest fat value. A study done by Hoke et al. (1999), observed the same trend in that an inverse relationship existed between moisture and fat content. In their study the highest fat and lowest moisture content was found to be in the raw lean rib and blade cuts. The loin also had the highest energy value of the three cuts, followed by the shoulder cut and then the leg with the lowest energy value. The same was found for the fatty acid profiles, where the leg cut contained the lowest fatty acid content of the three cuts, i.e. C16:0 (1.50 g/100 g), C18:0 $(0.88 \mathrm{~g} / 100 \mathrm{~g})$ saturated fatty acids, $\mathrm{C} 18: \ln 9 \mathrm{c}(2.14 \mathrm{~g} / 100 \mathrm{~g})$ monounsaturated fatty acid and $\mathrm{C} 18: 2 \mathrm{n} 6 \mathrm{c}(0.17 \mathrm{~g} / 100 \mathrm{~g})$ polyunsaturated fatty acid. Maturity of animals influences the meat fatty acid composition. Ono et al. (1984), studied lambs from two age groups and found that lambs from younger animals had higher amounts of some of the saturated fatty acids, and a higher polyunsaturated/saturated fatty acid ratio than those of older animals. 


\subsection{Cooked cuts}

The only significant differences between the cooked cuts (Table 4) were for the moisture, protein, fat, as well as for three minerals (magnesium, potassium and sodium). As expected, moisture losses due to cooking resulted in an increase in the protein and cholesterol concentration. The shoulder and leg had significantly more moisture than the loin cut. According to Lawrie (1998) it is feasible that significant differences may exist between specific muscle locations in the carcass or that breed and age has an effect. The essential amino acids may also differ at different parts of the animal (Lawrie, 1998). The loin cut $(27.79 \mathrm{~g} / 100 \mathrm{~g})$ had significantly $(p<0.001)$ more protein when compared to the $\operatorname{leg}(24.46 \mathrm{~g} / 100 \mathrm{~g})$ and shoulder cuts $(23.05 \mathrm{~g} / 100 \mathrm{~g})$. As expected, the shoulder had significantly more fat than the loin and leg cuts. A significant difference was found for ash content, with the loin cut having the highest ash content, followed by the shoulder cut.

Except for sodium, the cooked shoulder cut had the lowest mineral content of the three cuts, and the loin cut had the highest mineral content except for iron. The shoulder contained significantly less magnesium when compared to the loin and the leg. The loin had significantly more potassium with the shoulder the least. The leg cut had significantly lower sodium content, when compared to the loin cut. The loin cut had the highest zinc content, although not significantly, when compared to the leg and the shoulder. These results contrasted the findings of the study by Ono et al. (1984), where the blade chop (shoulder cut) showed the highest value for zinc and the loin chop the lowest. There were no significant differences in the nutrient values for the vitamins and fatty acids and cholesterol when comparing the three cooked cuts.

\subsection{Raw vs. cooked cuts}

Red meat contains an array of micronutrients, which are required for general heath and well-being with some being in substantial amounts. The nutrients showing the greatest differences between the treatments, were protein, total fat, as well as the C16:0, C18:0 and C20:0 saturated fatty acid and C18:1n9c monounsaturated fatty acid. As expected, moisture losses due to cooking resulted in an increase in the protein and cholesterol concentration. The present study found that the protein value (Table 5) was significantly higher in the cooked lamb shoulder, loin and leg cuts when compared to the raw shoulder, 
loin and leg cuts. Furthermore, the cooked loin cut contained the most protein $(27.79 \mathrm{~g} / 100 \mathrm{~g}$ edible portion) of the three cooked cuts. The total fat content ranged from $6.15 \mathrm{~g} / 100 \mathrm{~g}$ in the raw leg and $11.25 \mathrm{~g} / 100 \mathrm{~g}$ in the raw shoulder to between $7.67 \mathrm{~g} / 100 \mathrm{~g}$ in the cooked leg and $9.86 \mathrm{~g} / 100 \mathrm{~g}$ in the cooked shoulder cut. In this study the iron content differed, although not significantly, between the raw and cooked samples. Iron content decreased in the cooked shoulder and loin cuts but showed an increase in the cooked leg cut. The raw shoulder cut had $0.75 \mathrm{mg}$ iron/100 g. The same result was found for the raw loin $(0.99 \mathrm{mg}$ iron/100 g) when compared to the cooked loin cut $(0.62 \mathrm{mg}$ iron/100 g). However, the opposite was found for the cooked leg cut, where the raw leg contained $1.14 \mathrm{mg}$ iron/100 g iron compared to $1.20 \mathrm{mg} / 100 \mathrm{~g}$ iron in the cooked cut. Similar results were found by Lombardi-Boccia et al. (2002), where cooking of lamb cuts showed an increase in the iron content between raw and cooked samples. Furthermore, when iron values for raw lean lamb are compared with that of lean beef (11\% fat) (Schönfeldt et al., 1996), the raw loin lamb cuts analysed had a higher iron content than that of beef loin $(0.99 \mathrm{mg} / 100 \mathrm{~g}$ (lamb, loin) vs. $0.63 \mathrm{mg} / 100 \mathrm{~g}$ (A age beef loin with $11 \%$ fat). When cooked, the opposite was found in that cooked beef loin, contained more iron $(1.94 \mathrm{mg})$ per $100 \mathrm{~g}$ gram portion than cooked loin $(0.62 \mathrm{mg} / 100 \mathrm{~g}$ edible portion) from lamb (Schönfeldt et al 1996). Kruger et al. (2003), emphasised that red meats are the richest source of highly available iron in the diet because it occurs in the most bio-available form, namely haem iron, which is only found in food from animal origin. Lombardi-Boccia et al. (2005), also states that meat (animal products) has the highest bioavailability of iron in the form of haem iron.

The cooked shoulder cut contained the least vitamin $\mathrm{B}_{1}, \mathrm{~B}_{2}$ and $\mathrm{B}_{12}$ of the three cooked cuts, the cooked loin cut had the least vitamin $\mathrm{B}_{3}$ and the cooked leg the least vitamin $\mathrm{B}_{6}$. The vitamin content for the selected B vitamins of the three cooked lamb cuts were lower, although not significantly, than that of the raw cuts. The cooked shoulder cut, except for vitamin $\mathrm{B}_{3}$ and $\mathrm{B}_{6}$, contained the least vitamin $\mathrm{B}_{1}, \mathrm{~B}_{2}$ and vitamin $\mathrm{B}_{12}$ $(0.8 \mu \mathrm{g} / 100 \mathrm{~g})$, while the cooked loin cut contained the least vitamin $\mathrm{B}_{3}(1.25 \mathrm{mg} / 100 \mathrm{~g})$ and the cooked leg cut, the least vitamin $\mathrm{B}_{6}(0.10 \mathrm{mg} / 100 \mathrm{~g})$. This could be attributed to the fact that cooking influences the variability of water soluble vitamins in meat due to instability to heat or light and liquid (Lombardi-Boccia et al., 2005). However, when compared to other meat products, it is clear that lamb is a good source of the B vitamins, especially vitamin $\mathrm{B}_{12}$, as supported by Badiani et al. (1998). 
Cooking of the three cuts had a concentrating effect on all the nutrients, due to the associated decrease in moisture content during cooking. The only exception was for iron, which decreased in two cuts, namely the shoulder and loin cuts with increased iron content in the leg cut. These results are similar to those reported by Purchas et al. (2004), where a large proportion of the haem iron in beef semitendinosus muscle decreased during cooking.

The cooked shoulder and loin cuts mainly contained significantly less fatty acid than raw cuts while the cooked leg cut contained significantly more of the C14:0, C16:0 and C20:0 saturated fatty acids than that of the raw cut. The cholesterol content for all three cooked lamb cuts was higher than that of the raw cuts, with the shoulder cut containing the highest cholesterol. The cooked shoulder cut had the highest SFA (C14:0-0.59 g/100 g and $\mathrm{C} 16: 0-2.05 \mathrm{~g} / 100 \mathrm{~g})$ content except for C18:0 that was the lowest $(0.78 \mathrm{~g} / 100 \mathrm{~g})$ within the three samples. The same was found for the MUFA. The shoulder contained the highest $\mathrm{C} 16: 1$ and $\mathrm{C} 18: 1 \mathrm{n} 9 \mathrm{t}$ fatty acids with the least C18:1n9c MUFA as well as PUFA C18:2n6t and C18:2n6c. According to Lawrie (1998) it is feasible that significant differences may exist between specific muscle locations in the carcass. However, research in the UK revealed that in general, muscle from cattle, sheep and pigs is a valuable source of polyunsaturated fatty acids in the diet (Lawrie, 1998).

\subsection{Recommended dietary allowances}

The RDA for males, aged 25-30 (Whitney and Rolfes, 2002) were used as a reference point to evaluate the possible nutrient contribution of lamb, as determined during this study. The RDA provides a benchmark for estimating nutrient needs of healthy people which should be met by the consumption of a variety of nutrient rich food (Gericke, 2003). Three of the nutrients in cooked South African lamb provide more than a third of the RDA's for males aged 25-30 per 100 g edible portion (Table 6). A cooked shoulder, loin and leg lamb cuts provide on average 39\% protein, 37\% potassium and $38 \%$ vitamin $\mathrm{B}_{12}$ of RDA for this group of males. Energy from a $100 \mathrm{~g}$ portion provides $6.12 \%$ of the RDA. Lower levels of nutrients are included in the list namely vitamin $\mathrm{B}_{3}$ provides $11 \%$, zinc $11 \%$, vitamin $\mathrm{B}_{6} 6 \%$. iron $6 \%$, vitamin $\mathrm{B}_{2} 4 \%$, and vitamin $\mathrm{B}_{1}$ that represent $3 \%$ of the RDA. 
Table 6.

Contribution of $100 \mathrm{~g}$ of soft tissue from three cuts of cooked lamb ( $\pm 7 \% \mathrm{SCF})$ to the nutrient requirements (RDA values) of males, age $25-50$ years

\begin{tabular}{|c|c|c|c|c|c|c|c|c|c|}
\hline \multicolumn{10}{|c|}{ Contribution of 100 g edible portion lean lamb ( $\pm 7 \%$ SCF) to the RDA males $25-50$ years } \\
\hline Nutrients & $\begin{array}{l}\text { Un } \\
\text { it }\end{array}$ & $\begin{array}{l}\text { RDA } \\
\text { males } \\
\text { 25-50 }\end{array}$ & $\begin{array}{l}\text { Average } \\
\% \\
\text { contribu } \\
\text { tion }\end{array}$ & Shoulder & $\begin{array}{l}\text { Shoulder\% } \\
\text { contribution }\end{array}$ & Loin & $\begin{array}{l}\text { Loin \% } \\
\text { contribu } \\
\text { tion }\end{array}$ & Leg & Leg\% contribution \\
\hline \multicolumn{10}{|c|}{ Proximate analysis } \\
\hline Moisture & $\mathrm{g}$ & - & - & 66.8 & - & 63.49 & - & 66.03 & - \\
\hline $\begin{array}{l}\text { Protein } \\
(N \times 6.25)\end{array}$ & $\mathrm{g}$ & 63 & 39.8 & 23.1 & 36.6 & 27.8 & 44.1 & 24.5 & 38.8 \\
\hline Fat & $\mathrm{g}$ & - & - & 9.86 & - & 7.80 & - & 7.67 & - \\
\hline Ash & $\mathrm{g}$ & - & - & 0.95 & - & 1.19 & - & 1.05 & - \\
\hline $\begin{array}{l}\text { Food energy } \\
\text { (calculated) }\end{array}$ & $\mathrm{kJ}$ & 12180 & 6.12 & 757 & 6.22 & 755 & 6.20 & 723 & 5.94 \\
\hline \multicolumn{10}{|l|}{ Minerals } \\
\hline $\begin{array}{l}\text { Magnesium } \\
(\mathrm{Mg})\end{array}$ & $\mathrm{mg}$ & 420 & - & 18.0 & - & 24.2 & - & 22.9 & - \\
\hline Potassium (K) & $\mathrm{mg}$ & 800 & 37.3 & 261 & 32.6 & 330 & 41.25 & 303 & 37.9 \\
\hline Sodium $(\mathrm{Na})$ & $\mathrm{mg}$ & - & - & 68.9 & - & 83.3 & - & 61.8 & - \\
\hline Zinc $(\mathrm{Zn})$ & $\mathrm{mg}$ & 15 & 11.4 & 1.24 & 8.27 & 2.20 & 14.66 & 1.71 & 11.4 \\
\hline Iron $(\mathrm{Fe})$ & $\mathrm{mg}$ & 10 & 6.09 & - & - & 0.62 & 6.20 & 1.20 & 12.0 \\
\hline
\end{tabular}




\begin{tabular}{|c|c|c|c|c|c|c|c|c|c|}
\hline \multicolumn{10}{|c|}{ Contribution of 100 g edible portion lean lamb $( \pm 7 \%$ SCF) to the RDA males $25-50$ years } \\
\hline Nutrients & $\begin{array}{l}\text { Un } \\
\text { it }\end{array}$ & $\begin{array}{l}\text { RDA } \\
\text { males } \\
\mathbf{2 5 - 5 0 ^ { a }}\end{array}$ & $\begin{array}{l}\text { Average } \\
\% \\
\text { contribu } \\
\text { tion }\end{array}$ & Shoulder & $\begin{array}{l}\text { Shoulder\% } \\
\text { contribution }\end{array}$ & Loin & $\begin{array}{l}\text { Loin \% } \\
\text { contribu } \\
\text { tion }\end{array}$ & Leg & Leg\% contribution \\
\hline \multicolumn{10}{|l|}{ Vitamins } \\
\hline Thiamin $\left(\mathrm{B}_{1}\right)$ & $\mathrm{mg}$ & 1.2 & 3.47 & 0.03 & 2.08 & 0.04 & 3.50 & 0.06 & 4.83 \\
\hline $\begin{array}{l}\text { Riboflavin } \\
\left(\mathrm{B}_{2}\right)\end{array}$ & $\mathrm{mg}$ & 1.3 & 4.15 & 0.04 & 3.08 & 0.07 & 5.38 & 0.05 & 4.00 \\
\hline $\operatorname{Niacin}\left(\mathrm{B}_{3}\right)$ & $\mathrm{mg}$ & 16 & 11.7 & 1.37 & 8.56 & 1.25 & 7.81 & 1.63 & 10.2 \\
\hline $\begin{array}{l}\text { Pyridoxine } \\
\left(\mathrm{B}_{6}\right)\end{array}$ & $\mathrm{mg}$ & 1.7 & 6.80 & 0.12 & 6.94 & 0.132 & 7.76 & 0.10 & 5.71 \\
\hline $\begin{array}{l}\text { Cyanocobalam } \\
\text { in }\left(B_{12}\right)\end{array}$ & $\mu \mathrm{g}$ & 2.4 & 38.8 & 0.83 & 34.6 & 0.91 & 37.9 & 1.05 & 43.8 \\
\hline
\end{tabular}

${ }^{a}$ Whitney and Rolfes (2002), RDA for males 25-50 years. 


\subsection{Contribution of $100 \mathrm{~g}$ of cooked lamb to the nutrient requirements of males}

When the nutrient content of lean lamb $( \pm 7 \% \mathrm{SCF})$ is compared to that of beef, chicken and pork, the different species contained similar amounts of protein $(25.01-30.9 \mathrm{~g} / 100 \mathrm{~g})$, and also provides all the essential amino acids in proportions that meet nutrient requirements with cooked beef fillet (A age, 11\% fat) being the richer source of iron (3.32 mg/100 g), zinc $(4.20 \mathrm{mg} / 100 \mathrm{~g})$ and vitamin $\mathrm{B}_{12}(2.25 \mu \mathrm{g} / 100 \mathrm{~g})$ (Schönfeldt et al., 1996). According to Lawrie (1998), with respect to species differences, the high content of iron in cooked beef reflects the greater concentration of myoglobin in this species than in lamb or pork. The lean loin cut from lamb $( \pm 7 \% \mathrm{SCF})$ had the lowest iron content $(0.62 \mathrm{mg} / 100 \mathrm{~g})$ and cooked white meat of chicken (42 days old) the lowest vitamin $\mathrm{B}_{12}$ $(0.23 \mathrm{mg} / 100 \mathrm{~g})$ content according to Schönfeldt et al. (1998).

\subsection{Nutrient density}

Nutrient-dense foods are important sources of many essential nutrients including protein, iron, zinc, some vitamins and fatty acids. According to Whitney and Rolfes (2002), nutrient density is a measure of the nutrients a foodstuff provides relative to the energy it provides. The more nutrients and the fewer kiloJoules, the higher the nutrient density. The nutrient density of a food for a specific nutrient is calculated as follows: The nutrient in $100 \mathrm{~g}$ food $\times$ the RDA for energy divided by the energy in $100 \mathrm{~g}$ food. For example, to calculate the nutrient density of iron in $100 \mathrm{~g}$ cooked deboned meat (iron $2.35 \mathrm{~g}$, energy: $1097 \mathrm{~kJ}$ ) in the diet of males 25-50 years (RDA for iron: $10 \mathrm{mg}$ and energy: $12180 \mathrm{~kJ}$ )

\section{$(2.35 \mathrm{mg} / 1097 \mathrm{~kJ}) \times(12180 \mathrm{~kJ} / 10 \mathrm{mg})=2.6 \mathrm{l}$.}

A value exceeding 1 indicates a good source of that nutrient. In Table 7, the majority of the values are above 1, confirming that three of the cooked lamb cuts, supply significant quantities of a range of protein, iron, zinc and vitamin $\mathrm{B}_{12}$ for a limited amount of energy. 
Table 7.

Nutrient density of $100 \mathrm{~g}$ cooked, deboned South African lean lamb cuts $( \pm 7 \% \mathrm{SCF})$

\begin{tabular}{|l|l|l|l|}
\hline Nutrients & \multicolumn{3}{|l|}{$\mathbf{1 0 0}$ g edible portion of cooked lean lamb cuts $( \pm 7 \%$ SCF) } \\
\hline & Loin & Leg & Shoulder \\
\hline Protein & 7.11 & 6.54 & 5.88 \\
\hline Iron & 1.00 & 2.02 & 0.09 \\
\hline Zinc & 2.36 & 1.92 & 1.33 \\
\hline Vitamin $B_{12}$ & 6.11 & 7.37 & 5.56 \\
\hline
\end{tabular}

Nutrient density=Value $\geqslant 1.00$ : good source (Van Heerden, 2005).

\section{Conclusions}

It is evident from this study that lamb provides a variety of valuable nutrients required by human consumers. Another positive attribute of South African lamb is that the protein and mineral content increase during cooking due to moisture loss and that the iron (although it was found to be the lowest in this study), is 3-5 times more readily available than iron from plant foods.

Meat from the lean lamb $( \pm 7 \% \mathrm{SCF})$ can be regarded as an important dietary source of the B vitamins, although some may be lost due to leaching during the cooking process. Lamb is undoubtedly an excellent source of nutrients that are required for good health. The results showed that there is variation in the micronutrient content between raw and cooked treatments of the three different lamb cuts analysed especially for the proximate analyses values and the minerals of cooked cuts. Cooking affected mainly the moisture, protein, fat, ash and energy values, which were higher in the cooked meat cuts as well differed significantly between the cooked cuts. There was no significant difference in the iron and zinc between the cooked cuts. Furthermore, results showed differences in both trace elements and B vitamins among different cuts, although the differences were not statistically significant.

Lamb $( \pm 7 \%$ SCF $)$ makes a valuable contribution to the RDA for males, aged 25-50 years, with regard to the protein, potassium, zinc, vitamin $\mathrm{B}_{12}$ and $\mathrm{B}_{3}$ when included as part of a balanced meal plan. Therefore, as recommended in healthy eating advice around 
the world, lean meat especially lean lamb, consumed in moderation should be promoted as part of a healthy balanced diet.

Consuming nutrient-dense foods, as part of a balanced diet that includes the recommended servings, can help consumers achieve good health. Comparing data with other countries show that the use of non-local data sources can produce differences in the assessment of the nutrient composition and may cause conflicting interpretation of dietary intake. Therefore, accurate local data on nutrient composition is essential for assessing dietary intakes, determining the relationship between dietary intake and disease occurrence as well as for communicating nutrient information to the consumers. This study contributes valuable data to the nutrient profile of red meat, especially South African lamb. However, this is data on A2 class only ( $\pm 7 \% \mathrm{SCF})$, and more work is required on the rest of the classification system of South African lamb to ensure a complete data set.

\section{References}

American Meat Science Association, 1995 American Meat Science Association, Research Guidelines for Cookery, Sensory Evaluation and Instrumental Tenderness Measurements of Fresh Meat, American Meat Science Association and National Live Stock and Meat Board, Chicago, IL (1995).

Association of Official Analytical Chemists International (AOAC), 2005 Association of Official Analytical Chemists International (AOAC), 2005. Official methods of analysis, of AOAC International, eighteenth ed. Maryland, USA.

Badiani et al., 1998 A. Badiani, N. Nanni, P.P. Gatta, F. Bitossi, B. Tolomelli and M. Manfredini, Nutrient content and retention in selected roasted cuts from 3-month-old ram lambs, Food Chemistry 61 (1/2) (1998), pp. 89-100.

Chan et al., 1996 W. Chan, J. Brown, S.M. Church and D.H. Buss, Meat Products and Dishes, Supplement to McCance \& Widdowson's The Food Composition of Foods, The Royal Society of Chemistry, London, Cambridge (1996).

Christopherson and Glass, 1969 S.W. Christopherson and R.L. Glass, Preparation of milk fat methyl esters by alcoholysis in an essentially non-alcoholic solution, Journal of Dairy Science 52 (1969), pp. 1289-1290. 
Dionex System 20001, 1988 Dionex System 20001, 1988. Operation and Maintenance Manual. Dionex Document 1991. No 034341, Dionex Corp., Sunnyvale, CA, USA. Draft Regulations Relating to the Labeling and Advertising of Foodstuffs as part of the Foodstuffs and Cosmetics and Disinfectants Act, 1972 Draft Regulations Relating to the Labeling and Advertising of Foodstuffs as part of the Foodstuffs, Cosmetics and Disinfectants Act, 1972, published in August 2002 to become law in 2005 http://0www.doh.gov.za.innopac.up.ac.za:80/department/dir_foodcontr.html $/$. Enser, 2000 Enser, M., 2000. Producing meat for healthy eating. In: Proceedings of the 46th International Congress of Meat Science and Technology, Argentinia, 27 August-1 Septembe, p. 124.

Fellman et al., 1992 J.K. Fellman, W.E. Artz, P.D. Tassinari, C.L. Cole and J. Augustin, Simultaneous determination of thiamin and riboflavin in selected foods by highperformance liquid chromatography, Journal of Food Science 47 (1992), pp. 2048-2050. Gebhardt and Thomas, 2002 Gebhardt, S.E., Thomas, R.G., 2002. Nutrient value of foods. US Department of Agriculture, Agricultural Research Service, Nutrient Data Laboratory, Beltsville, MD (Home and Garden Bulletin 72:58). GenStat for Windows, 2003 GenStat for Windows, 2003. Release 4.2. fifth ed. VSN International Ltd., Oxford, UK.

Gericke, 2003 Gericke, G., 2003. Better eating for better health: principles and practices of planning a healthful diet. Unit 4, In Graduate Readings Volume 3. Fundamentals of Nutrition Security in Rural Development, pp. 227-244.

Greenfield and Southgate, 2003 H. Greenfield and D.A.T. Southgate, Food Composition Data, Food and Agriculture Organization of the United Nations, Rome (2003). Hoke et al., 1999 I.M. Hoke, D.R. Buege, W. Ellefson and E. Maly, Nutrient and related food composition of exported Australian lamb cuts, Journal of Food Composition and Analysis 12 (1999), pp. 97-109.

International Dairy Federation, 1993 International Dairy Federation, 1993. Determination of the Total Nitrogen content of Milk. IDF standard 20 B IDF, Brussels. International Dairy Federation, 1996 International Dairy Federation, 1996. Determination of Fat Content. Röse Gottlieb. Gravimetric Method. IDF standard 20 B IDF, Brussels. 
Kirton et al., 1962 A.H. Kirton, R.A. Barton and A.L. Rae, The efficiency of determining the chemical composition of lamb carcass, Journal of Agricultural Science, Cambridge 58 (1962), pp. 381-386.

Kruger et al., 2003 Kruger, R., van der Spuy, E.H., Viljoen, A.T., 2003. Nutrition in the rural context Unit 5, In Graduate Readings Volume 3. Fundamentals of Nutrition Security in Rural Development, p. 344.

Latham, 1997 M.C. Latham, Human Nutrition in the Developing World, FAO, Rome (1997).

Lawrie, 1998 R.A. Lawrie, Meat Science (sixth ed), Woodhead Publishing Limited, Cambridge, England (1998).

Lewis et al., 1995 Lewis, J., Milligan, G., Hurt, A., 1995. NUTTAB95—nutrient data table for use in Australia. Food Standards Australia New Zealand, vol. 1. Commonwealth of Australia.

Lombardi-Boccia et al., 2002 G. Lombardi-Boccia, B. Martinez-Dominguez and A. Aguzzi, Total heme and non-heme iron in raw and cooked meats, Journal of Food Science 67:5 (2002), pp. 1738-1741.

Lombardi-Boccia et al., 2005 G. Lombardi-Boccia, S. Lanzi and A. Aguzzi, Aspects of meat quality: trace elements and B vitamins in raw and cooked meats, Journal of Food Composition and Analysis 18 (2005), pp. 39-46.

McCance and Widdowson, 1940 R.A. McCance and E.M. Widdowson, The chemical composition of foods. Medical Research Council Special Report Series No. 235, His Majesty's Stationery Office, London (1940).

National Department of Agriculture, 1990 National Department of Agriculture, 1990. Agricultural Product Standards Act, 1990 Regulations regarding the classification and marketing of meat. ACT No. 119 of 1990:9-14.

Ono et al., 1984 K. Ono, B.W. Berry, H.K. Johnson, E. Russek, C.F. Parker, V.R. Cahill and P.G. Althouse, Nutrient composition of lamb of two age groups, Journal of Food Science 49 (1984), pp. 1233-1257.

Purchas et al., 2004 R.W. Purchas, S.M. Rutherfurd, P.D. Pearce, R. Vather and B.H.P. Wilkinson, Cooking temperature effects on the forms of iron and levels of several other components in beef semitendinosus muscle, Meat Science 68 (2004), pp. 201-207. 
Sayed et al., 1998 N. Sayed, Y. Frans and H.C. Schönfeldt, Composition of South African foods: milk \& milk products, eggs, meat \& meat products. Supplement to the MRC Food Composition Tables 1991. SAFCOD, Medical Research Council, UDMS, Cape Town (1998).

Schönfeldt, 1998 Schönfeldt, H.C., 1998. Effect of age on beef quality. Ph.D. Thesis, University of Pretoria.

Schönfeldt et al., 1996 H.C. Schönfeldt, R.E. Visser, J.M. van Niekerk and S.M. van Heerden, The Nutritional Content of South African beef., South African Meat Board, Pretoria (1996) ISBN 0-620-20379X.

Schönfeldt et al., 1998 Schönfeldt, H.C., van Heerden S.M., van Niekerk, J.M., Visser, R.E., Heinze P.H., 1998. The nutrient content of South African fresh and frozen chicken. ISBN0-620-22682X.

Smuts et al., 1992 C.M. Smuts, M. Kruger, P.J. van Jaarsveld, J.E. Fincham, R. Schall, K.J. van der Merwe and A.J.S. Benadé, Postaglandins, Leukotrienes Essential Fatty Acids 47 (1992), pp. 129-138.

Snedecor and Cochran, 1980 G.W. Snedecor and W.G. Cochran, Statistical Methods (seventh ed), Iowa State University Press (1980) pp. 234-235.

South African Food Composition Data (SAFCoD), 2000 South African Food Composition Data (SAFCoD), 2000. Brochure. MRC Nutrition Intervention Programme, PO Box 19070, Tygerberg, 7505, South Africa.

Steyn et al., 2000 Steyn, N., MacIntyre, U., Labadarios, D., Maunder, E., Swart, R., Nesamvuni, A.E., Gericke, G., Huskisson, J., Vorster, H.H., Dannhauser, A. 2000. The food and nutrient intakes of children aged 1-9 years in South Africa: The National Food Consumption Survey. In: Proceedings 6th Biennial Nutrition Congress 2000, From Lab to Land, South Africa, 16-18 August.

United Stated Department of Agriculture, 1989 United Stated Department of Agriculture, 1989. Composition of Foods: Lamb, Veal and Game Products. Agricultural Handbook number 8-17.

Van Heerden, 2005 Van Heerden, S.M., 2005. The nutrient composition of SA lamb. Ph.D. Thesis, unpublished. 
Whitney and Rolfes, 2002 E.N. Whitney and S.R. Rolfes, Understanding Nutrition (ninth ed), Wadsworth, London (2002).

Corresponding author. Tel.: +27 12672 6212; fax: +27 126651551 . 